\title{
The Milky Way, the Galactic Halo, and the Halos of Galaxies
}

\author{
Ortwin Gerhard
}

Max-Planck-Institut für Extraterrestrische Physik, Postsach 1312, 85741 Garching, Germany

\begin{abstract}
The Milky Way, "our" Galaxy, is currently the subject of intense study with many ground-based surveys, in anticipation of upcoming results from the Gaia mission. From this work we have been learning about the full three-dimensional structure of the Galactic box/peanut bulge, the distribution of stars in the bar and disk, and the many streams and substructures in the Galactic halo. The data indicate that a large fraction of the Galactic halo has been accreted from outside. Similarly, in many external galaxy halos there is now evidence for tidal streams and accretion of satellites. To study these features requires exquisite, deep photometry and spectroscopy. These observations illustrate how galaxy halos are still growing, and sometimes can be used to "time" the accretion events. In comparison with cosmological simulations, the structure of galaxy halos gives us a vivid illustration of the hierarchical nature of our Universe.
\end{abstract}

Keywords. Galaxies: general, Milky Way, halos, formation, kinematics and dynamics, structure.

\section{Introduction: the Milky Way as a galaxy}

Our Galaxy and its satellites together with the similarly massive Andromeda galaxy and its satellite system constitute the Local Group, a loose galaxy group in the outer reaches of the Virgo cluster. The Milky Way is a barred spiral galaxy with stellar mass $M_{*} \simeq 6 \times 10^{10} M_{\odot}$, near the massive end of the distribution (Lange et al. 2015). It has relatively low gas content and star formation rate, which places it in the green valley of the distribution of similar galaxies found in the SDSS survey (Licquia \& Newman 2015). The central bulge is dominated by a box/peanut structure showing that most of the bulge formed from the disk through dynamical evolution processes. Together with the embedded disk the stellar mass in the bulge region is $\sim 1.6 \times 10^{10} M_{\odot}$, nearly $30 \%$ of the total (Portail et al. 2015). The box/peanut bulge is the three-dimensional part of the Galactic bar, which extends outwards in the disk to $R \sim 5 \mathrm{kpc}$ and has a mass of order additional $10^{10} \mathrm{M}_{\odot}$ (Wegg et al. 2015). The Galactic stellar disk includes almost all of the remaining stellar mass. Its dynamical structure is well-studied in the solar neighbourhood, located at $\simeq 8.2 \mathrm{kpc}$ from the center, but its radial scale-length, while relatively short, $2-3 \mathrm{kpc}$, is not accurately known. Beyond the solar radius, the outer disk has a prominent warp. The disk is surrounded by a halo of old, low-metallicity stars with only $\sim 1 \%$ of the stellar mass, and some 50 satellite galaxies including the prominent Magellanic clouds and Sagittarius dwarf. See Bland-Hawthorn \& Gerhard (2016) for a more extensive discussion and additional references.

\section{The Galactic halo - observations and predictions}

The Milky Way's stellar halo was first identified as a population of old, high-velocity, metal poor stars near the Sun, similar to the stars in globular clusters. The halo stars showed large random motions, little if any rotation, and a spheroidal to spherical spatial distribution. Following the influential paper of Eggen et al. (1962), a classical view of the halo developed as a smooth envelope of ancient stars from the time when the Galaxy first 
collapsed. Today, thanks to large stellar surveys such as SDSS, the stellar halo is seen as a complex structure with multiple components and unrelaxed substructures, which still accretes matter in the form of smaller galaxies. These are then tidally disrupted in the gravitational field, as is well-illustrated in the famous field of streams (Belokurov et al. 2006).

This complex structure is consistent with hierarchical galaxy formation models which predict that the Milky Way should have accreted of order 100 satellite galaxies. Irregular density distributions are predicted in the outer halo due to shells and tidal streams from the satellite galaxies disrupting in the tidal field. At those radii, the time required for stars to complete multiple orbits and spread throughout the halo volume is a significant fraction of the age of the Galaxy. In simulations, the majority of the halo is often built at early times, $\sim 10$ Gyr ago, and most of the stellar halo stars come from the disruption of one or a few massive satellites accreted early-on. The outer halo is built more recently than the inner halo and the halo properties evolve as it builds up by the accretion (Bullock \& Johnston 2005; Cooper et al. 2010). Some of the inner halo may have formed in situ, i.e., within the main body of the Galaxy (Abadi et al. 2006). Recent simulations suggest that a fraction of stars formed in the early Galactic disk could have been ejected into the inner halo, and further in situ halo stars could have formed from gas stripped from infalling satellites (McCarthy et al. 2012; Pillepich et al. 2015). Observational evidence for different properties of the inner and outer halo is presented by Carollo et al. (2007); Beers et al. (2012).

Various samples of tracer stars with photometric distances show that the overall density profile of the stellar halo steepens at $r \sim 25 \mathrm{kpc}$. The data favour a double power law model, with an inner slope $\alpha_{\text {in }} \simeq-2.5$ and an outer slope $\alpha_{\text {out }} \simeq-(3.7-5.0)$, depending somewhat on the tracers used (Faccioli et al. 2014; Pila-Díez et al. 2015). The halo is slightly flattened, with axial ratio increasing from $q_{\text {in }} \simeq 0.65$ to $q_{\text {out }} \simeq 0.8$. Studies of a large number of stars with radial velocities and proper motions find that the stellar velocity ellipsoid of nearby halo stars is aligned with spherical coordinates, and quite strongly radially anistropic (Smith et al. 2009; Bond et al. 2010). The radial velocity dispersion profile falls quickly from a local $\sigma_{r}=140 \mathrm{~km} \mathrm{~s}^{-1}$ to $\sigma_{r}=100 \mathrm{~km} \mathrm{~s}^{-1}$ at $r \sim 20 \mathrm{kpc}$, then remains approximately flat to $R \sim 50 \mathrm{kpc}$, and finally decreases to $\sigma_{r} \sim 35 \mathrm{~km} \mathrm{~s}^{-1}$ at $\sim 100 \mathrm{kpc}$ (Battaglia et al. 2005; Kafle et al. 2014), suggesting perhaps a tidal truncation at the largest radii.

From the density profile and kinematics of these tracer stars the mass of the Milky Way's dark matter halo can be determined. Other methods involve the kinematics of globular clusters and dwarf galaxies, estimation of the local escape velocity, and modelling of halo streams. Overall, the resulting mass of the Galaxy is still quite uncertain; in BlandHawthorn \& Gerhard (2016) we estimate $M_{\mathrm{vir}}=1.3 \pm 0.3 \times 10^{12} \mathrm{M}_{\odot}$ from the various studies.

In the stellar density maps, many streams, tails, and overdensities are seen. The four largest structures are the Sgr stream, the Galactic anticentre structure, the Virgo cloud, and the Hercules-Aquila cloud. Together these structures have a mass of $2-3 \times 10^{8} M_{\odot}$ (Belokurov et al. 2013). The Sgr stream alone provides $\sim 20 \%$ of all the halo debris, including probably $\sim 20$ globular clusters. Bell et al. (2008) found with SDSS main sequence turn-off stars that the rms deviation of the star counts around a smooth model is $\sim 40 \%$ of the model itself, suggesting that a large fraction of the halo stars are in substructures. However, using blue horizontal branch stars as tracers, Deason et al. (2011) find a lower ratio, $\sim 5-20 \%$. The total stellar mass in the halo is estimated $M_{s}=4-7 \times 10^{8} M_{\odot}$ (Bland-Hawthorn \& Gerhard 2016), about one percent of the Galaxy's stellar mass.

In simulations of stellar halos such as the Aquarius suite (Cooper et al. 2010), a significant galaxy-to-galaxy variance in the halo properties is observed, which is due to the 
different accretion histories of the simulated galaxies. Thus a full picture of the halo properties requires studying external galaxies.

\section{Stellar halos in nearby galaxies}

Deep images of stellar halos in spiral galaxies (Martínez-Delgado et al. 2010) show a variety of features indicating accretion of satellite galaxy stars, such as streams, spikes, wedges, giant plumes, partially disrupted satellites, umbrella-shaped tidal debris and other tidal clouds. With deep imaging it is possible to detect emission down to $\mu_{V} \sim 27-29$ mag $\operatorname{arcsec}^{-2}$ (see van Dokkum et al. 2014), however, various uncertainties due to flat fielding, sky background, bright star halos, or Galactic cirrus need to be controlled. Spectroscopy is generally possible only to $\mu_{V} \sim 25.5 \mathrm{mag} \operatorname{arcsec}^{-2}$, so information on the accreted stellar population is limited to colours, and kinematics is often only available in the inner halos.

For the nearest galaxies where resolved stars can be observed $(\sim 5 \mathrm{Mpc}$ from the ground, $\sim 12 \mathrm{Mpc}$ from space), one can probe to very deep surface brightness levels $\left(\mu_{V} \sim 33\right.$ mag $\left.\operatorname{arcsec}^{-2}\right)$, constrain the stellar population from colour-magnitude diagrams, and obtain kinematics for bright stars. A beautiful example is the Pandas survey of Andromeda (M31), showing that the M31 halo is dominated by debris substructure (e.g., Ferguson et al. 2002). Combining a large number of fields out to $200 \mathrm{kpc}$ radius, Ibata et al. (2014) constructed stellar density and metallicity maps. These show an outward gradient in the overall metallicity of the M31 halo, a strong dependence of substructure on metallicity, and an ancient, low-metallicity $([\mathrm{Fe} / \mathrm{H}]<-1.7)$, approximately smooth, and near-spherical halo component.

Results from the GHOSTS survey of nearby spiral galaxies with the Hubble Space Telescope were recently published by Monachesi et al. (2016). In these more distant galaxies, information is generally restricted to a number of fields along the major and minor axes of the galaxy, reaching distances of typically $\sim 50 \mathrm{kpc}$ from the galaxy center. The color profiles determined from these data show considerable diversity; field-to-field variations indicate stellar population variations such as would be expected if the halos had been built from several diverse accreted objects. The color and inferred metallicity profiles can be flat or falling, without clear correlation with galaxy stellar mass or rotation velocity, consistent with the galaxy-to-galaxy scatter predicted by simulations. By comparison with this sample of 6 galaxies, the Milky Way halo has a low metallicity and a weak gradient, while the M31 halo has high metallicity and a prominent gradient.

The seminal deep imaging study of Mihos et al. (2005) of the extended halos of earlytype galaxies (ETGs) in the Virgo cluster core has been extended to larger galaxy samples by Tal et al. (2009) and Duc et al. (2015). The deep surface brightness maps show a variety of streams, shells, and other tidal features at large radii, indicating on-going accretion also in these more massive galaxies. Simulations such as those of Cooper et al. (2013) using particle-tagging methods predict that the fraction of accreted stars in galaxies is a strong function of mass, ranging from a few percent for Milky Way galaxies to 80-90\% in the most massive ETGs. Indeed, the stellar halo in the Milky Way is a minor component by mass, but in giant galaxies like M87 in the Virgo cluster, almost all stars may have been accreted.

This is related to the observed size evolution of massive galaxies between $z=2$ and the present epoch (Trujillo et al. 2007; van Dokkum et al. 2010). The latter authors construct stacked surface density profiles for samples of galaxies at constant number density to show that the profiles grow more extended with decreasing redshift, from Sersic index $n \simeq 2$ at $z=2$ to $n \simeq 6$ at $z=0$, corresponding to an increase in the effective radius by a factor 
4-5. The size evolution is believed to be driven by minor merger driven accretion (Naab et al. 2009; Oser et al. 2010).

In the outer halos kinematic measurements are possible only for bright tracers, planetary nebulae (PNs) and globular clusters (GCs). PNs are direct descendants of halo stars and generally follow light; they reproduce stellar kinematics in regions of overlap (Coccato et al. 2009). For very old, metal-rich stellar populations however the specific PN-frequency can be 2-3 times lower (Longobardi et al. 2015a). GCs split into red and blue populations, where the blue GCs trace halo and dwarf galaxy light, and the red GCs bulge light. The kinematics of red GCs is often similar to stellar kinematics (Pota et al. 2013) but not always (Coccato et al. 2013). In M31 the outer halo GCs lie preferentially on streams but are underrepresented on the diffuse halo light (Veljanoski et al. 2014). Thus PNs and GCs appear to trace somewhat different modes of accretion.

A tantalizing case of accretion has recently been uncovered in the Virgo-central ETG M87. In the projected phase-space of some 300 PNs Longobardi et al. (2015b) found a chevron structure extending to a radius of $\simeq 90 \mathrm{kpc}$ where both sides of the chevron meet at the systemic velocity. Reexamination of deep images from Mihos et al. (2005) revealed a giant crown-like substructure extending over $20 \mathrm{kpc} \times 60 \mathrm{kpc}$ just inside this radius, which consists of bluer stars than the main M87 halo, and locally increases the halo surface brightness by $\sim 60 \%$. From the PN kinematics the satellite appears to have come in on a near-radial orbit extending across much of the galaxy, about $\sim 1 \mathrm{Gyr}$ ago (based on simulations by Weil et al. 1997), such that the crown is located in the region where the debris orbits reach apocenter. This is direct evidence that the M87 outer halo is presently growing by accretion, and suggests that beyond $r \sim 70 \mathrm{kpc}$ it is no longer in dynamical equilibrium. A somewhat similar chevron was found in the GCs by Romanowsky et al. (2012); this appears to reach to larger radii and no photometric correspondent has been found, so that the relation to the crown remains unclear. At yet larger radii several streamers are seen in the deep image, apparently falling into M87; these are too faint for PNs but GCs have been found on one. Beyond about $r \sim 50 \mathrm{kpc}$ the $\mathrm{PN}$ velocity distribution shows an additional kinematic component with velocity dispersion $\sim 1000 \mathrm{~km} \mathrm{~s}^{-1}$. This intracluster component has a much shallower density profile than the M87 halo proper, and dominates beyond $r \sim 150 \mathrm{kpc}$ (Longobardi et al. 2015a).

PNs and GCs have been independently used to determine the angular momentum and mass distributions in ETG halos. $\lambda_{R}$-profiles from the PN.S survey (Arnaboldi et al. in prep.) show that ETGs which are slow rotators at small radii also rotate slowly in their halos, whereas fast rotators can have rising, constant, and sometimes falling $\lambda_{R^{-}}$ halo profiles (Coccato et al. 2009). GCs from the SLUGGS project (Brodie et al. 2014) show very similar $\lambda_{R}$-profiles (Pota et al. 2013), and both are in good agreement with predicted $\lambda_{R}$-profiles from cosmological resimulations of ETG halos (Wu et al. 2014). Dynamical modelling of the halo kinematic data has shown that dark matter dominates beyond $\sim 5 R_{e}$ (de Lorenzi et al. 2008; Morganti et al. 2013; Cappellari et al. 2015), again in good agreement with the simulated ETGs.

Stellar population studies from integrated light show metallicity gradients to $\sim 2.5 R_{e}$, consistent with the accretion of old, metal-poor stars into the halos of ETGs (Greene et al. 2012). In the halo of the central galaxy NGC 3311 in the Hydra I cluster, dissolution of two satellite galaxies is on-going (Arnaboldi et al. 2012), and deep spectra show that the accreted dwarf galaxy HCG 026 indeed adds very old and metal-poor stars to the BCG halo (Coccato et al. 2011). Such studies will be greatly advanced by deep integral-field spectroscopy with the planned E-ELT and TMT giant telescopes. 


\section{Conclusions}

The study of galaxy halos is challenging because of the faint surface brightnesses in the halos, but offers much interesting insight into the late accretion and formation histories of galaxies. In the Milky Way halo streams, tails, and substructures show the importance of satellite accretion as predicted by hierarchical galaxy formation models. Smooth spatial and velocity structures in the inner halo indicate efficient mixing, while the steep outer density profile and declining velocity dispersion lead to a relatively low virial mass. In the near future, we expect substantial progress based on the astrometric data currently assembled by the Gaia mission. From the distance and proper motion measurements for halo stars it will be possible to study stellar streams in the halo in much greater detail than before and obtain unprecedented constraints on the Galaxy's accretion history and halo mass distribution.

The Milky Way is a massive spiral galaxy in a relatively isolated environment, going through the late stages of its evolution. It has low gas content and star formation rate, and a prominent box/peanut bulge and bar in the disk reaching $5 \mathrm{kpc}$ radius. Simulations predict significant cosmic variance in galaxy accretion histories and stellar halo structure even at constant dark matter mass. Studies of external galaxy halos have confirmed some of this variance, e.g., in the difference between the Milky Way and M31 halos, and have revealed signatures of accretion at faint surface brightness around both spiral and elliptical galaxies. In massive galaxies, accreted halos are predicted to dominate over in situ stars, consistent with the size evolution observed in luminous ETGs. The few accreted satellites studied in ETG halos have been found to be old and metal-poor. Much work remains to be done to obtain a more complete picture of the accretion histories in galaxy halos and of the properties of the accreted satellites and their stars.

\section{References}

Abadi, M. G., Navarro, J. F., \& Steinmetz, M. 2006, MNRAS, 365, 747

Arnaboldi, M., et al. 2012, Astron. Astrophys., 545, A37

Battaglia, G., et al. 2005, MNRAS, 364, 433

Beers, T. C., et al. 2012, Ap. J., 746, 34

Bell, E. F., et al. 2008, Ap. J., 680, 295

Belokurov, V., et al. 2006, Ap. J., 642, L137

—. 2013, MNRAS, 437, 116

Bland-Hawthorn, J. \& Gerhard, O. 2016, ARAA, eprint arXiv:1602.07702

Bond, N. A., et al. 2010, Ap. J., 716, 1

Brodie, J. P., et al. 2014, Ap. J., 796, 52

Bullock, J. S. \& Johnston, K. V. 2005, Ap. J., 635, 931

Cappellari, M., et al. 2015, Ap. J., 804, L21

Carollo, D., et al. 2007, Nature, 450, 1020

Coccato, L., Arnaboldi, M., \& Gerhard, O. 2013, MNRAS, 436, 1322

Coccato, L., Gerhard, O., Arnaboldi, M., \& Ventimiglia, G. 2011, Astron. Astrophys., 533, A138

Coccato, L., et al. 2009, MNRAS, 394, 1249

Cooper, A. P., et al. 2010, MNRAS, 406, 744

-. 2013, MNRAS, 434, 3348

de Lorenzi, F., et al. 2008, MNRAS, 385, 1729

Deason, A. J., Belokurov, V., \& Evans, N. W. 2011, MNRAS, 416, 2903

Duc, P.-A., et al. 2015, MNRAS, 446, 120

Eggen, O. J., Lynden-Bell, D., \& Sandage, A. R. 1962, Ap. J., 136, 748

Faccioli, L., et al. 2014, Ap. J., 788, 105

Ferguson, A. M. N., et al. 2002, Astron. J., 124, 1452 
Greene, J. E., et al. 2012, Ap. J., 750, 32

Ibata, R. A., et al. 2014, Ap. J., 780, 128

Kafle, P. R., Sharma, S., Lewis, G. F., \& Bland-Hawthorn, J. 2014, Ap. J., 794, 59

Lange, R., et al. 2015, MNRAS, 447, 2603

Licquia, T. C. \& Newman, J. A. 2015, Ap. J., 806, 96

Longobardi, A., Arnaboldi, M., Gerhard, O., \& Hanuschik, R. 2015a, Astron. Astrophys., 579, A135

Longobardi, A., Arnaboldi, M., Gerhard, O., \& Mihos, J. C. 2015b, Astron. Astrophys., 579, L3

Martínez-Delgado, D., et al. 2010, Astron. J., 140, 962

McCarthy, I. G., et al. 2012, MNRAS, 420, 2245

Mihos, J. C., Harding, P., Feldmeier, J., \& Morrison, H. 2005, Ap. J., 631, L41

Monachesi, A., et al. 2016, MNRAS, 457, 1419

Morganti, L., et al. 2013, MNRAS, 431, 3570

Naab, T., Johansson, P. H., \& Ostriker, J. P. 2009, Ap. J., 699, L178

Oser, L., et al. 2010, Ap. J., 725, 2312

Pila-Díez, B., et al. 2015, Astron. Astrophys., 579, A38

Pillepich, A., Madau, P., \& Mayer, L. 2015, Ap. J., 799, 184

Portail, M., Wegg, C., Gerhard, O., \& Martinez-Valpuesta, I. 2015, MNRAS, 448, 713

Pota, V., et al. 2013, MNRAS, 428, 389

Romanowsky, A. J., et al. 2012, Ap. J., 748, 29

Smith, M. C., et al. 2009, MNRAS, 399, 1223

Tal, T., van Dokkum, P. G., Nelan, J., \& Bezanson, R. 2009, Astron. J., 138, 1417

Trujillo, I., et al. 2007, MNRAS, 382, 109

van Dokkum, P. G., Abraham, R., \& Merritt, A. 2014, Ap. J., 782, L24

van Dokkum, P. G., et al. 2010, Ap. J., 709, 1018

Veljanoski, J., et al. 2014, MNRAS, 442, 2929

Wegg, C., Gerhard, O., \& Portail, M. 2015, MNRAS, 450, 4050

Weil, M. L., Bland-Hawthorn, J., \& Malin, D. F. 1997, Ap. J., 490, 664

$\mathrm{Wu}, \mathrm{X}$., et al. 2014, MNRAS, 438, 2701 